\title{
Design and Simulation of a Low-Voltage Low-Offset Operational Amplifier
}

\author{
Babak Gholami \\ Department of Electrical Engineering \\ Kazeroon Branch, Islamic Azad University \\ Kazeroon, Iran
}

\begin{abstract}
In many applications, offset of the OP-AMPs should be canceled to high accuracy be accomplished. In this work, an asymmetrical differential input circuit with active DC offset rejection circuit was implemented to minimize the systematic offset of the amplifier. The proposed OP-AMPs show that the systematic offset voltages is less than $80 \mu \mathrm{V}$.
\end{abstract}

Keywords — component; formatting; style; styling; insert

\section{INTRODUCTION}

The CMOS Op-Amp is an important building block of linear and switched-capacitor circuits. However, mismatch of the devices causes an offset voltage, which limits the highprecision application. Commonly, the offset sources of OPAMPs are categorized as systematic offset and random offset. The systematic offset happens because of the channel length modulation of transistors and the value of the offset voltages are the functions of the input and output common mode voltages [1, 2, 3]. For example, in the formal OP-AMPs, the channel length modulation of transistor shows the systematic offset. However, the channel length modulation is unimportant, with no feedback, the output common mode voltage ever shows the fixed voltage level and doesn't follow the change of the input common mode voltage level. This difference between the input and output common mode level shows very small systematic offset voltages. The systematic offset can be minimized by controlling bias current of input stage to sustain the input and output common mode in same level.

The general method of offset cancelation of OP-AMPs is the feedback-capacitor circuit as shown in Fig.1 [7]. At first, as the switch 1 and 2 are turned on, the offset voltage is stored in $\mathrm{C}$ offset. Then the offset of $\mathrm{V}_{\text {OUT }}$ is omitted when the switch 1 and 2 are turned off and the switch 3 and 4 are turned on. But this circuit has some disadvantages of large capacitor, and many CMOS switches which is the source of the switching error. In this work, a continuous time asymmetrical differential input circuit with common mode feedback circuit which can minimize the offset of OP-AMPs is presented.

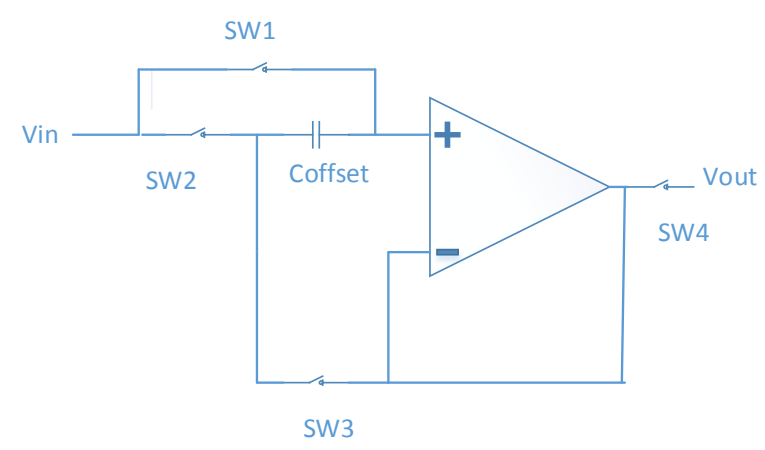

Fig. 1. Typical Offset Compensation Circuit

\section{CIRCUIT DESCRIPTION}

The proposed OP-AMP is composed of three parts: Asymmetrical differential input stage, Common Mode Feedback (CMFB) stage and output stage. In input stage, there are cross-coupled input devices, $\mathrm{M}_{1}, \mathrm{M}_{2}, \mathrm{M}_{3}$ and $\mathrm{M}_{4}$ and tail current sources, $\mathrm{M}_{5}$ and $\mathrm{M}_{6}$. Input devices have asymmetrical differential structure. It means that W/L of $M_{1}$ and $M_{3}$ is larger than that of $\mathrm{M}_{2}$ and $\mathrm{M}_{4}$, so the transconductance $\left(\mathrm{g}_{\mathrm{m}}\right)$ of $M_{1}$ and $M_{3}$ is larger than that of $M_{2}$ and $M_{4}$. The proposed CMFB circuit is shown in figure 3 . The first stage is the combination of $M_{9}-M_{12}$ and current sources $M_{14}-M_{17}[10,8]$.

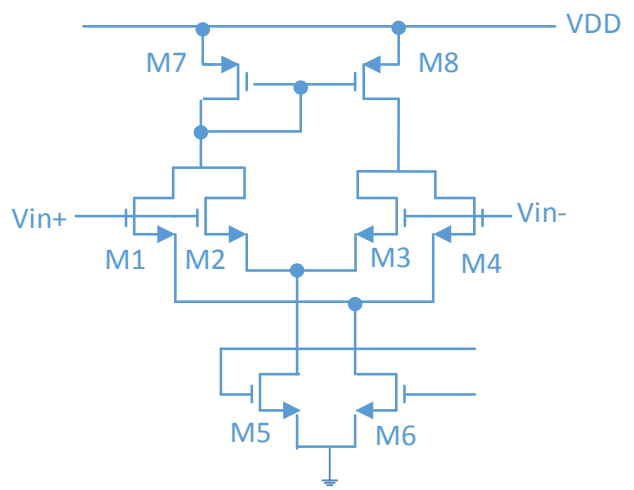

Fig. 2. Input Stage 
The second stage is the combination of $\mathrm{M}_{14}-\mathrm{M}_{19}$. Long channel NMOS transistors are used for input stage to minimize the differential pair nonlinearity and to insert more input voltage swing. They also minimize the $\mathrm{V}_{\text {bias }}$ caused by the transistor mismatch among $\mathrm{M}_{9}-\mathrm{M}_{12}$. The common-mode level of the input and the output could be detected and amplified by the DC offset rejection circuit, and changed to the feedback signal for current sinks of the amplifier. This is a negative feedback network.

By adjusting the current of the current sinks, the input and the output common-mode voltage would be maintained in same level to minimize the systematic offset. The difference between input and output common-mode level will be amplified. In the proposed circuit, the sum of drain currents, $\mathrm{I}_{\mathrm{M} 5}$ and $\mathrm{I}_{\mathrm{M} 6}$ is constant. Therefore, small signal differential mode open loop voltage gain of input stage is given by:

$A_{\text {vd,input-stage }}=-\left(g_{m(M 3)}+g_{m(M 4)}\right) \cdot\left(r_{o(M 8)}\right.$ II $\left.r_{o(M 3)} I I r_{o(M 4)}\right)$

Where $g_{m}$ is the transconductance and $r_{0}$ is the output resistance of transistor. The total small signal open loop differential mode voltage gains are given by:

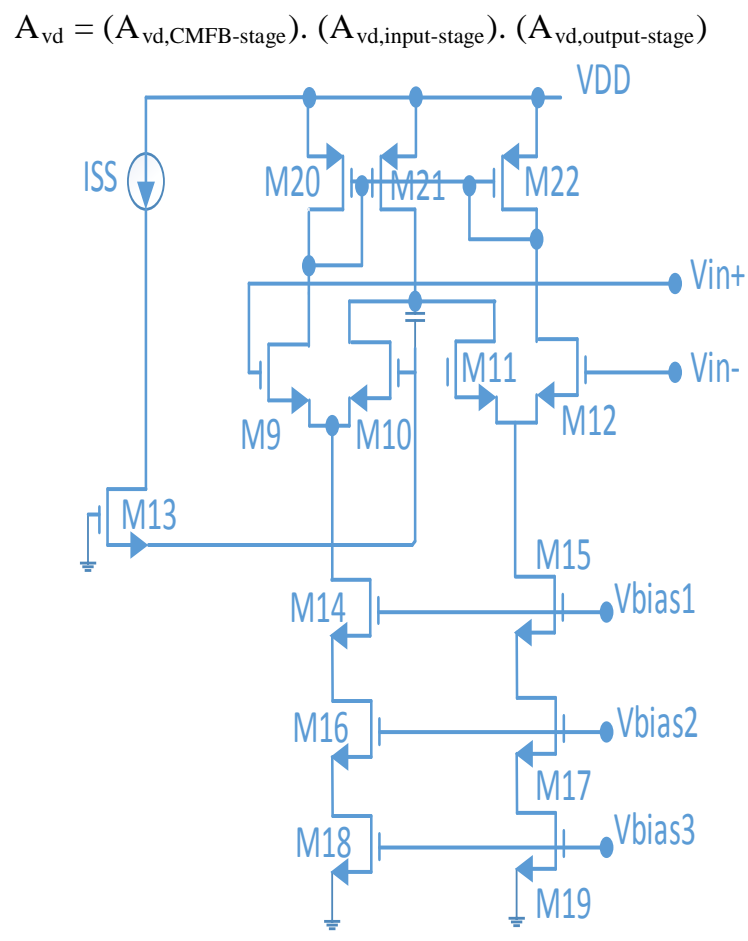

Fig. 3. DC Offset Rejection Circuit

\section{OUtPut STAGE}

The class-AB output stage shown in Figure 4, is composed of an output buffer. Transistor $\mathrm{M}_{23}-\mathrm{M}_{26}$ form two floating current sources to provide bias current of branches, which confirm the transistor $\mathrm{M}_{33}, \mathrm{M}_{34}, \mathrm{M}_{35}$ and $\mathrm{M}_{36}$ work in saturation region. Their gate voltages are provided by two biasing branches respectively. The current signal $\mathrm{I}_{\mathrm{IN} 1}$ and $\mathrm{I}_{\mathrm{IN} 2}$ are subtracted through current mirror and amplified by pushpull stage $[10,2]$.

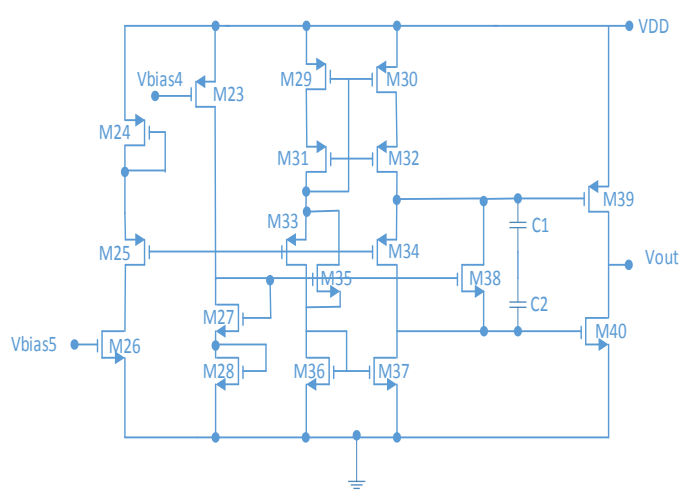

Fig. 4. Output Circuit

\section{SimUlation CiRCUIT}

Figure 5 shows the AC responses of the proposed OPAMP while driving a $2 \mathrm{pF}$ load. It shows $60 \mathrm{~dB}$ open-loop gain, $63.5^{\circ}$ phase margin, and $2.82 \mathrm{MHz}$ unity gain bandwidth. DC sweep analysis of the amplifier connected in an inverting unity-gain configuration is shown in figure 6 . The simulation results showed good following characteristic between Vin and Vout, and the offset voltage less than $80 \mu \mathrm{V}$ by averaging.

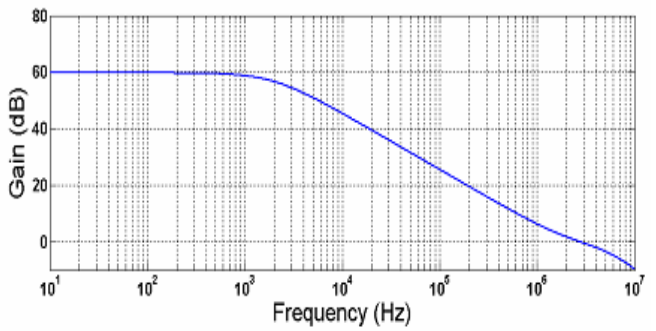

Fig. 5(a)

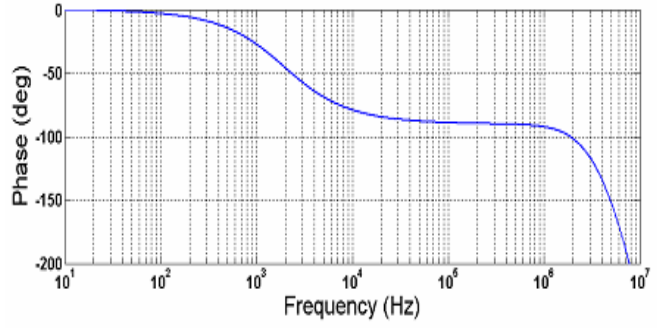

Fig. 5(b)

Fig. 5. Simulation Result of Frequency Response: (a) Magnitude, (b) Phase

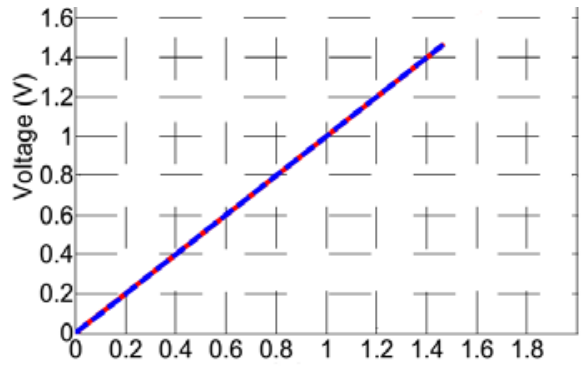

Fig. 6. The Simulation Result of the Following Characteristic 


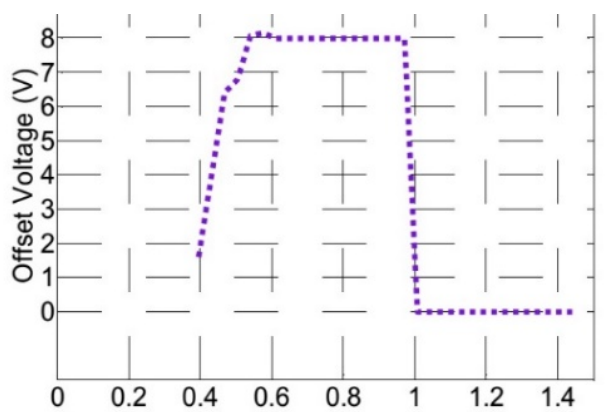

Fig. 7. The Simulation Result of the Offset Tunning Range

TABLE I. PERFORMANCE SUMMARY

\begin{tabular}{|l|l|}
\hline CMOS Technology & $0.18 \mu \mathrm{m}$ \\
\hline Supply & $1.5 \mathrm{~V}$ \\
\hline Gain & $60 \mathrm{~dB}$ \\
\hline Phase Margin & $62^{\circ}$ \\
\hline Unity Gain Bandwidth & $2.73 \mathrm{MHz}$ \\
\hline Input Offset Voltage & $80 \mu \mathrm{V}$ \\
\hline
\end{tabular}

\section{CONCLUSION}

An offset cancellation technique that uses an asymmetrical differential input circuit with active DC offset rejection circuit has been presented. Simulation results show that Op-Amp offset voltage is less than $80 \mu \mathrm{V}$ in entire operating voltage range.

\section{ACKNOWLEDGEMENT}

We gratefully acknowledge the support and comments from the editor and the reviewers, respectively. This work is in part supported by Islamic Azad University- Kazeroon Branch.

\section{REFERENCES}

[1] B. Razavi, Design of Analog CMOS Integrated Circuits, McGraw-Hill, 2002, pp.291-336.

[2] D. A. Johns and K. Martin, Analog Integrated Circuit Design, New York: John Wiley \& Sons, Inc., 1997, pp.221-251.

[3] J. H. Huijsing, R. Hogervorst and K. J. de Langen, "Low power low voltage VLSI operational amplifiers cells", IEEE Transactions on Circuits and Systems I, vol. 42, pp.841-852, Nov. 1995.

[4] J. Y. Zhang, L. Wang and B. Li, "Design of Low Offset Low Power CMOS Amplifier for Biosensor Application”, J Biomed Sci Eng, vol. 2, pp.538-542, 2009.

[5] K. T. Lim, S. J. Kim and O. K. Kwon, "The OP-Amplifier with offset cancelation circuit”, IEEE, pp.445-447, 2003.

[6] P. E Allen and D. R Holberg, CMOS Analog Circuit Design, Second Edition, New York, Oxford University Press Inc., 2002, pp.352-415.

[7] P. Kakoty, "Design of a high frequency low voltage CMOS operational amplifier”, Int. j. VLSI des. commun. Syst, vol. 2, pp.3680-3692, March 2011.

[8] R. Jacob Baker, CMOS Circuit Design, Layout and Simulation, 2nd ed, Wiley-IEEE Press, 2004, pp.863-904.

[9] S. Franco, Design with Operational Amplifiers and Analog Integrated Circuits, McGraw-Hill College, 1988, pp.60-92.

[10] S. Sheikhaei, S. Mirabbasi, and A. Ivanov, "A 0.35m CMOS Comparator Circuit For High-Speed ADC Applications”, IEEE, vol. 6, pp.6134 - 6137,2005.

[11] Y. Tsividis, Operation and Modeling of the MOS Transistor, 2nd ed, Boston, MA: McGraw-Hill, 1998, pp.123-154. 